\title{
Mixture of Functional Linear Models and Its Application to $\mathrm{CO}_{2}$-GDP Functional Data
}

\author{
Shaoli Wanga ${ }^{\mathrm{a}}$, Mian Huang ${ }^{\mathrm{a}}$, Xing $\mathbf{W u}^{\mathrm{a}}$, Weixin Yao ${ }^{\mathrm{b}}$ \\ ${ }^{a}$ School of Statistics and Management and Key Laboratory of Mathematical \\ Economics at SHUFE, Ministry of Education, Shanghai University of Finance and \\ Economics (SHUFE), Shanghai 200433, P. R. China \\ ${ }^{b}$ Department of Statistics, University of California, Riverside, CA, 92521, U.S.A.
}

\begin{abstract}
Functional linear models are important tools for studying the relationship between functional response and covariates. However, if subjects come from an inhomogeneous population that demonstrates different linear relationship between the response and covariates among different subpopulations/clusters, a single functional linear model is no longer adequate for the data. A new class of mixtures of functional linear models for the analysis of heterogeneous functional data is introduced. Identifiability is established for the proposed class of mixture models under mild conditions. The proposed estimation procedures combine the ideas of local kernel regression, functional principal component analysis and EM algorithm. A generalized likelihood ratio test based on a conditional bootstrap is given as to whether the regression coefficient functions are constant. A Monte Carlo simulation study is conducted to examine the finite sample performance of the new methodology. Finally, the analysis of $\mathrm{CO}_{2}$-GDP data reveals the dynamic patterns of relationship between $\mathrm{CO}_{2}$ and GDP among different countries.
\end{abstract}

Keywords: Mixtures of functional linear regressions; Identifiability; EM-type algorithm; Kernel regression; Functional principal component analysis; Conditional bootstrap; Hypothesis test. 


\section{Introduction}

The observations of functional data are functions defined over some set $T$. The last two decades have seen tremendous advances in functional data analysis. Ramsay and Silverman $(2002,2005)$ offer an excellent introduction to functional data analysis. Ferraty and Vieu (2006) gives a detailed account of nonparametric methods for functional data. Horváth and Kokoszka (2012) focuses on statistical inference for functional data, particulary on hypothesis tests in various functional data settings. Ferraty and Romain (2011) contains a comprehensive and up-to-date review on a broad range of topics in functional data analysis. Bongiorno et al. (2014) brings in the recent advances in functional data analysis and related areas. Bosq (2000) and Bosq and Blanke (2007) lay the mathematical foundations for functional data analysis.

In regression analysis for functional data, either the response or covariates, or both can be functions. Functional linear models (FLMs) (Ramsay and Silverman, 2005) are useful for modeling the linear relationship between a scalar response and functional covariates. However, FLMs fail for nonlinear regression functions, and nonparametric techniques have been employed in the literature. Ferraty et al. (2013) introduces the functional projection pursuit regression. Chen et al. (2011) studies single and multiple index functional regression models with nonparametric link functions. Kudraszow and Vieu (2013) proposes a kNN generalized regression estimator for the regression function and proves its uniform consistency. When both multivariate and functional covariates are present, semi-functional partial linear regression models can be used (Aneiros-Pérez and Vieu, 2006).

When both the response and covariates are functions, we can use a specific class of FLMs, called concurrent FLMs:

$$
y(t)=\boldsymbol{X}(t)^{T} \boldsymbol{\beta}(t)+\varepsilon(t), \quad t \in T,
$$

where $y(t)$ is a univariate response process, $\boldsymbol{X}(t)$ a $p$-dimensional covariate process, 
$\boldsymbol{\beta}(t)$ the unknown smooth regression coefficient function, and $\varepsilon(t)$ a zero mean error process that is independent of $\boldsymbol{X}(t)$. The time set $T$ is typically assumed to be a closed bounded interval. In the concurrent model (1.1), the functional response $y(t)$ depends on the functional covariates $\boldsymbol{X}(t)$ in a point-wise manner. This type of FLMs has drawn an increasing attention recently in the functional and longitudinal data analysis. Hoover et al. (1998) studies the estimation of the regression coefficient functions using the smoothing spline and the local polynomial regression. Fan and Zhang (2000) proposes a two-step kernel smoothing procedure for the data collected at the same scheduled time points for each subject. Eubank et al. (2004) developes Bayesian prediction intervals via the smoothing spline for the coefficient curves. Yao et al. (2005) studies a special case of the model (1.1), the mean functional model, for the irregular and sparse longitudinal data. To study the possible nonlinear relationship rather than model (1.1), Ferraty et al. (2012) takes a fully nonparametric approach.

The FLMs are useful for the functional data when all subjects obey the same linear relationship between the response and covariates. However, in some applications, the subjects might come from an inhomogeneous population which consists of several homogenous subpopulations/clusters, but within each subpopulation/cluster the concurrent linear model still holds. For this type of applications, a single FLM (1.1) is no longer adequate for modeling the functional data. This motivates us to extend the FLM (1.1) to heterogeneous functional data and propose a new class of functional regression models, called mixtures of FLMs. We will explain in more detail the new model in the next section.

The motivation for our new model also comes from the analysis of a $\mathrm{CO}_{2}$-GDP dataset. The $\mathrm{CO}_{2}$-GDP dataset contains two related variables for 175 countries for the years 1980-2005, the $\mathrm{CO}_{2}$ emissions per capita and the GDP per capita. It is of our interest to model how the $\mathrm{CO}_{2}$ emissions level depends on the GDP. Figure 1(a) depicts the $\mathrm{CO}_{2}$ emission curves of the 175 countries. Figure 1(b) shows a scatter 
plot of the cross-sectional subset of the data for the year 2005, along with two fitted regression lines, which demonstrates two different economic development paths among 175 countries. The slope for each subgroup gives the average associated increment of $\mathrm{CO}_{2}$ emissions per capita given an unit increment in GDP per capita (Huang and Yao, 2012). Most developed countries are from the lower component which has a smaller slope, and the representatives include the United States, the United Kingdom, Canada, Australia, etc. Representative countries from the top component, which has a bigger slope, include Kuwait, Saudi Arabia, Qatar, etc. For the original functional data, it is tempting to fit a two-component mixture of linear regressions to the crosssectional subset of each year, and check whether the slopes of two components vary over time. However, this naive fitting-one-mixture-a-year procedure suffers serious drawbacks. First, the naive procedure can not ensure a consistent labeling for each country across different years. Second, the slope estimates might change rapidly from year to year, since all slopes are estimated separately for different years. Third, it neglects any possible within-subject correlations across years for each country. Thus, a novel estimation procedure needs to be developed for the analysis of the functional $\mathrm{CO}_{2}$-GDP data to incorporate the information across different years, which is the main purpose of this paper. Our analysis of $\mathrm{CO}_{2}$-GDP data is an application of functional data modeling in the study of climate change. Functional data analysis has found a broad range of applications, such as economics, biomedicine, geology, environmetrics, paleoclimatology, etc. For example, a nonparametric functional regression model is applied to analyze and forecast the maximum ozone concentration (Aneiros-Pérez et al., 2004), bivariate splines are employed to study the ozone concentration forecasting (Ettinger et al., 2012). Both applications have a real response and functional covariates.

In this article, we propose a mixture of FLMs for heterogeneous functional data. The regression coefficients and covariances for each component are assumed to be smooth functions of $t$. We first establish the identifiability result for the proposed 


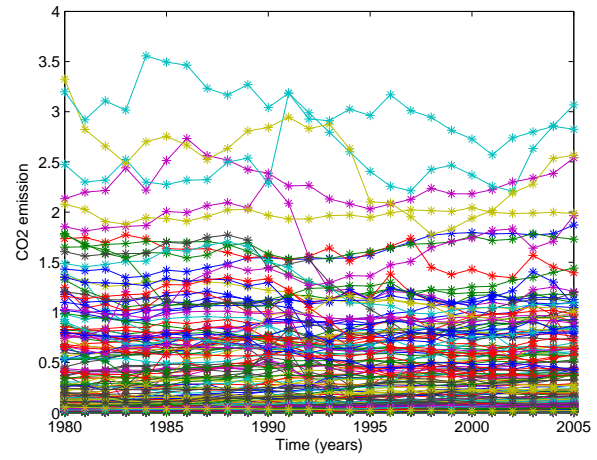

(a)

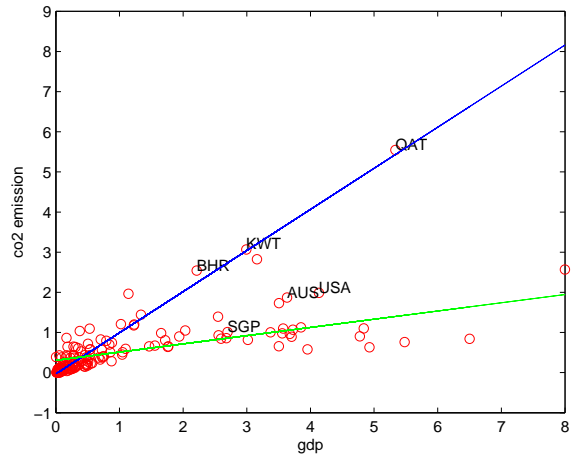

(b)

Figure 1: (a) Observed $\mathrm{CO}_{2}$ emission trajectories of 175 countries; (b) Scatter plot of $\mathrm{CO}_{2}$-GDP sub-dataset of year 2005 with two fitted regression lines. y: $\mathrm{CO}_{2}$ emission per capita; x: GDP per capita.

mixture model under mild conditions. To the best of our knowledge, this is the first identifiability result for mixtures of FLMs so far. We next develop an estimation procedure for the regression coefficients and covariance functions by combining the techniques of the kernel regression, the functional principal component analysis, and the EM algorithm. To choose the number of components for the mixture of FLMs based on the traditionally used information criteria, such as BIC and AIC, we propose an effective degree of freedom for both nonparametric regression coefficients and nonparametric covariance functions by adapting the idea of Fan et al. (2001). The Wilk's type of phenomenon (Fan et al., 2001) is also investigated for the model inference, and a conditional bootstrap method is proposed for the standard error estimation and the hypothesis testing. Finally, we examine the performance of the proposed estimation procedure and the bootstrap method empirically via a Monte Carlo simulation study and an application of the $\mathrm{CO}_{2}$-GDP data.

The rest of the paper is organized as follows. In Section 2, we introduce the mixture of functional linear models and prove its identifiability. In addition, we develop an estimation procedure by combining the techniques of kernel regression, functional principal component analysis, and EM algorithm. Model selection and the 
inference are studied in Section 3. Simulation results and a real data application are presented in Section 4. In Section 5, we provide some concluding remarks and discussions. Technical proofs are given in the Appendix.

\section{Mixtures of Functional Linear Models}

\subsection{Model Specification and Identifiability}

In this section we begin with the formulation of the mixture of FLMs. Let $\mathcal{C}$ be a latent class variable with a discrete distribution $P(\mathcal{C}=c)=\pi_{c}$, for $c=1,2, \cdots, C$. We assume that the number of components $C$ is known for the time being, and will discuss how to select $C$ adaptively in the next section. Conditioning on $\mathcal{C}=c$, $\{y(t), t \in T\}$ follows a functional linear model

$$
\left.y(t)\right|_{\mathcal{C}=c}=\mathbf{X}(t)^{T} \boldsymbol{\beta}_{c}(t)+\varepsilon_{c}(t)
$$

where $\mathbf{X}(t)$ is a $p$-dimensional random covariate process, $\boldsymbol{\beta}_{c}(t)$ is an unknown smooth regression coefficient function for the $c$ th component, and $\varepsilon_{c}(t)$ is a zero mean Gaussian process that is independent of $\mathbf{X}(t)$. We further assume that the error process $\varepsilon_{c}(t)$ consists of two parts, a trajectory effect $\zeta_{c}(t)$ and a measurement error effect $e(t)$ :

$$
\varepsilon_{c}(t)=\zeta_{c}(t)+e(t)
$$

The trajectory process $\zeta_{c}(t)$ is independent of $e(t)$ and has a covariance function $\Gamma_{c}(s, t)=\operatorname{Cov}\left\{\zeta_{c}(s), \zeta_{c}(t)\right\}$, which is a positive definite smooth function of $s$ and $t$; and the measurement error $e(t)$ is an uncorrelated process with a constant variance function $\sigma^{2}(t) \equiv \sigma^{2}$. The unconditional model of $\{y(t): t \in T\}$, without knowing the latent variable $\mathcal{C}$, is referred to as the mixture of functional linear models.

When there are no real covariates in the data, and the error processes are Gaussian, the mixture model (2.1) reduces to mixtures of Gaussian processes, which are also known as functional clustering models (James and Sugar, 2003; Luan and Li, 2003; Heard et al., 2006; Ma and Zhong, 2008). Huang et al. (2014) imposes smooth 
structures for both mean and covariance functions in the mixture of Gaussian processes, and developes estimation procedures based on the kernel regression. For the functional data with real covariates, Yao et al. (2011) proposes a mixture of nonconcurrent FLMs. Lu and Song (2012) studies a mixture of varying coefficient models for the longitudinal data analysis.

Identifiability is of fundamental importance for traditional parametric mixture models, since the parameter estimation and the inference must be based on identifiable models. For a classical finite mixture of parametric models, the identifiability issue is studied in Titterington et al. (1985). Next, we will first give a formal definition of the identifiability for mixtures of FLMs and then prove that the mixture of FLMs is identifiable under mild conditions. To best of our knowledge, this is the first identifiability result for mixtures of FLMs so far.

Definition 1. The mixture of functional linear models (2.1) is said to be identifiable if it does not admit another representation, i.e., if there is another latent variable $\mathcal{G}$, $P(\mathcal{G}=g)=\lambda_{g}, g=1, \ldots, G$, such that given $\mathcal{G}=g,\{Y(t), t \in T\}$ follows a Gaussian process with mean function $\mathbf{X}(t)^{T} \boldsymbol{\gamma}_{g}(t)$ and covariance function $\operatorname{Cov}\{Y(s), Y(t)\}=$ $\Omega_{g}(s, t)$, then $G=C$ and

$$
\lambda_{g}=\pi_{g}, \quad \gamma_{g}(t)=\boldsymbol{\beta}_{g}(t), \quad \Omega_{g}(s, t)=\Gamma_{g}(s, t), \quad s, t \in T, \quad g=1, \ldots, C,
$$

up to the permutation of component labels.

Theorem 1. Suppose that for each $t \in T$, the domain of $\mathbf{X}(t)$ contains an open set, and that for any $c=1, \ldots, C, \Gamma_{c}(s, t)$ is a positive definite and bivariate smooth function of $s$ and $t$, and that $\boldsymbol{\beta}_{c}(t)$ is a smooth function of $t$. Let $\mathbf{S}=\{t \in T$ : $\left(\boldsymbol{\beta}_{i}(t), \Gamma_{i}(t, t)\right)=\left(\boldsymbol{\beta}_{j}(t), \Gamma_{j}(t, t)\right)$ for some $\left.i \neq j, 1 \leq i, j \leq C\right\}$. If the set $T \backslash \mathbf{S}$ is not empty, then the above proposed mixture of FLMs is identifiable.

Based on the above Theorem, the mixture of FLMs is identifiable under some mild conditions. The proof is relegated to the Appendix. 


\subsection{Estimation Procedure}

By the well-known Karhunen-Loève theorem (Sapatnekar, 2011), conditioning on $\mathcal{C}=c$, an observed curve $\left\{y_{i}(t), \mathbf{X}_{i}(t)\right\}$ can be represented as

$$
y_{i}(t)=\mathbf{X}_{i}(t)^{T} \boldsymbol{\beta}_{c}(t)+\sum_{q=1}^{\infty} \xi_{i q c} v_{q c}(t)+e_{i}(t),
$$

where $v_{q c}(\cdot)$ s are eigenfunctions of the covariance function $\Gamma_{c}(s, t)$ with corresponding eigenvalues $\lambda_{q c} \mathrm{~s}$, and $\xi_{i q c} \mathrm{~s}$ are uncorrelated functional principal component (FPC) scores of the trajectories $\zeta_{c}(t)$ satisfying $\mathrm{E}\left(\xi_{i q c}\right)=0, \operatorname{Var}\left(\xi_{i q c}\right)=\lambda_{q c}, \lambda_{1 c} \geq \lambda_{2 c} \geq \ldots$, and $\sum_{q=1}^{\infty} \lambda_{q c}<\infty$.

Suppose subject $y_{i}(t)$ is observed at time $t_{i j}, j=1, \cdots, N_{i}$. We define the notation $y_{i j}=y_{i}\left(t_{i j}\right)$ for the simplicity, and similarly define notations $\varepsilon_{c i j}, e_{i j}$, etc. Based on (4.1), conditioning on $\mathcal{C}=c$, the observations $y_{i j}, j=1, \cdots, N_{i}$ and $i=1, \cdots, n$, can be written as

$$
y_{i j}=\mathbf{X}_{i}\left(t_{i j}\right)^{T} \boldsymbol{\beta}_{c}\left(t_{i j}\right)+\sum_{q=1}^{\infty} \xi_{i q c} v_{q c}\left(t_{i j}\right)+e_{i j},
$$

where $e_{i j}$ s are independent and identically distributed as $N\left(0, \sigma^{2}\right)$.

Next, we introduce two procedures for estimating the $\pi_{c}, \boldsymbol{\beta}_{c}(\cdot)$, and the covariance structure. In the first estimation procedure, we pretend that the observations are uncorrelated by ignoring the correlation structure among the data. The idea of using working independence correlation structure has been traditionally used by generalized estimating equation in the longitudinal data analysis (Liang and Zeger, 1986; Lin and Carroll, 2000). Let $\sigma_{c}^{* 2}(t)=\Gamma_{c}(t, t)+\sigma^{2}$. It follows that

$$
y_{i j}=\mathbf{X}_{i}\left(t_{i j}\right)^{T} \boldsymbol{\beta}_{c}\left(t_{i j}\right)+\varepsilon_{i j}^{*},
$$

where $\varepsilon_{i j}^{*}$ are independent errors satisfying $E\left(\varepsilon_{i j}^{*}\right)=0$ and $\operatorname{Var}\left(\varepsilon_{i j}^{*}\right)=\sigma_{c}^{* 2}\left(t_{i j}\right)$. Hence, 
$y_{i j}$ can be considered coming from the following mixture of Gaussian process:

$$
y(t) \sim \sum_{c=1}^{C} \pi_{c} N\left\{\mathbf{X}(t)^{T} \boldsymbol{\beta}_{c}(t), \sigma_{c}^{* 2}(t)\right\}
$$

Let $\phi\left(y \mid \mu, \sigma^{2}\right)$ be the density function of $N\left(\mu, \sigma^{2}\right)$. The log-likelihood function of $(2.5)$ is

$$
\sum_{i=1}^{n} \log \left[\sum_{c=1}^{C} \pi_{c} \prod_{j=1}^{N_{i}} \phi\left\{y_{i j} \mid \mathbf{X}_{i}\left(t_{i j}\right)^{T} \boldsymbol{\beta}_{c}\left(t_{i j}\right), \sigma_{c}^{* 2}\left(t_{i j}\right)\right\}\right] .
$$

We next introduce an EM-type estimation procedure to estimate the $\pi_{c}, \boldsymbol{\beta}_{c}(\cdot)$, and $\sigma_{c}^{* 2}(\cdot)$. The derivation of the procedure is given in the Appendix.

Estimation procedure 1 (Working Independence Covariance Structure)

1. Initial Value: For the pooled data, fit a $C$-component mixture of linear regression models with constant proportions and variances and obtain the estimates $\overline{\boldsymbol{\beta}}_{c}, \bar{\sigma}_{c}^{2}$ and $\bar{\pi}_{c}$. Set the initial values $\boldsymbol{\beta}_{c}^{(1)}(t)=\overline{\boldsymbol{\beta}}_{c}, \sigma_{c}^{2(1)}(t)=\bar{\sigma}_{c}^{2}$, and $\pi_{c}^{(1)}=\bar{\pi}_{c}$, $c=1, \ldots, C$.

2. E-step: For $i=1, \ldots, n$, and $c=1, \ldots, C$, calculate

$$
r_{i c}^{(l+1)}=\frac{\pi_{c}^{(l)}\left[\prod_{j=1}^{N_{i}} \phi\left\{y_{i j} \mid X_{i}\left(t_{i j}\right)^{T} \boldsymbol{\beta}_{c}^{(l)}\left(t_{i j}\right), \sigma_{c}^{* 2(l)}\left(t_{i j}\right)\right\}\right]}{\sum_{c=1}^{C} \pi_{c}^{(l)}\left[\prod_{j=1}^{N_{i}} \phi\left\{y_{i j} \mid X_{i}\left(t_{i j}\right)^{T} \boldsymbol{\beta}_{c}^{(l)}\left(t_{i j}\right), \sigma_{c}^{* 2(l)}\left(t_{i j}\right)\right\}\right]} .
$$

3. M-step: Update the component proportions by

$$
\pi_{c}^{(l+1)}=\frac{1}{n} \sum_{i=1}^{n} r_{i c}^{(l+1)} .
$$

For any time $t_{0}$ from a set of grid points $\left\{u_{1}, \ldots, u_{n_{\text {grid }}}\right\}$, update the slope and 
variance functions by

$$
\begin{aligned}
\boldsymbol{\beta}_{c}^{(l+1)}\left(t_{0}\right) & =\left\{\sum_{i=1}^{n} \mathbf{X}_{i}^{T} W_{i c}^{(l+1)}\left(t_{0}\right) \mathbf{X}_{i}\right\}^{-1}\left\{\sum_{i=1}^{n} \mathbf{X}_{i}^{T} W_{i c}^{(l+1)}\left(t_{0}\right) \mathbf{y}_{i}\right\}, \\
\sigma_{c}^{* 2(l+1)}\left(t_{0}\right) & =\frac{\sum_{i=1}^{n} \sum_{j=1}^{N_{i}} w_{c i j}^{(l+1)}\left\{y_{i j}-X_{i}\left(t_{i j}\right)^{T} \boldsymbol{\beta}_{c}^{(l+1)}\left(t_{0}\right)\right\}^{2}}{\sum_{i=1}^{n} \sum_{j=1}^{N_{i}} w_{c i j}^{(l+1)}},
\end{aligned}
$$

where $\mathbf{y}_{i}=\left(y_{i 1}, \cdots, y_{i N_{i}}\right)^{T}, \mathbf{X}_{i}=\left(\mathbf{X}_{i}\left(t_{i 1}\right), \ldots, \mathbf{X}_{i}\left(t_{i N_{i}}\right)\right)^{T}, w_{c i j}^{(l+1)}=r_{i c}^{(l+1)} K_{h_{\beta}}\left(t_{i j}-\right.$ $\left.t_{0}\right), W_{i c}^{(l+1)}\left(t_{0}\right)=\operatorname{diag}\left\{r_{i c}^{(l+1)} K_{h_{\beta}}\left(t_{i 1}-t_{0}\right), \ldots, r_{i c}^{(l+1)} K_{h_{\beta}}\left(t_{i N_{i}}-t_{0}\right)\right\}, K_{h}(\cdot) \equiv$ $h^{-1} K(\cdot / h), K(\cdot)$ is a kernel density, and $h_{\beta}$ is a bandwidth parameter.

4. Iteratively update the E-step and the M-step with $l=2,3, \ldots$, until the algorithm converges. Denote the resulting estimates of $\pi_{c}, \boldsymbol{\beta}_{c}(\cdot)$, and $\sigma_{c}^{* 2}(\cdot)$ by $\hat{\pi}_{c}$, $\hat{\boldsymbol{\beta}}_{c}(\cdot)$, and $\hat{\sigma}_{c}^{* 2}(\cdot)$, respectively. Denote the resulting posterior probability as $\hat{r}_{i c}$.

Note that the above estimate does not use the correlation information among the data and thus might lose some efficiency. Next, we introduce an improved estimation procedure which can incorporate the covariance functions into the model estimation by combining the techniques of the kernel regression, the functional principal component analysis (PCA), and the EM algorithm.

Given the estimates from Estimation procedure 1, the covariance function $\Gamma_{c}(s, t)$ could be estimated by a two-dimensional kernel smoother, which is to minimize

$$
\sum_{i=1}^{n} \hat{r}_{i c} \sum_{1 \leq j \neq l \leq N_{i}}\left[\hat{\gamma}_{i c}\left(t_{i j}, t_{i l}\right)-\beta_{0}\right]^{2} K_{h_{\Gamma}}\left(t_{i j}-s\right) K_{h_{\Gamma}}\left(t_{i l}-t\right),
$$

with respect to $\beta_{0}$, where $\hat{\gamma}_{i c}\left(t_{i j}, t_{i l}\right)=\left\{y_{i j}-\mathbf{X}_{i}\left(t_{i j}\right)^{T} \hat{\boldsymbol{\beta}}_{c}\left(t_{i j}\right)\right\}\left\{y_{i l}-\mathbf{X}_{i}\left(t_{i l}\right)^{T} \hat{\boldsymbol{\beta}}_{c}\left(t_{i l}\right)\right\}$, and $h_{\Gamma}$ is a bandwidth parameter for the covariance smoothing. If we can estimate the $\xi_{i q c}$ and $v_{q c}(\cdot)$ in $(2.3)$ from the estimated covariance function $\hat{\Gamma}_{c}(s, t)$, then we can transfer the correlated data into uncorrelated ones based on (2.3). The estimates 
of eigenvalues $\hat{\lambda}_{q c}$ and eigenfunctions $\hat{v}_{q c}(\cdot)$ are determined by the equations

$$
\int_{T} \hat{\Gamma}_{c}(s, t) \hat{v}_{q c}(s) d s=\hat{\lambda}_{q c} \hat{v}_{q c}(t)
$$

where $\hat{v}_{q c}(t)$ satisfies $\int_{T} \hat{v}_{q c}^{2}(t) d t=1$, and $\int_{T} \hat{v}_{p c}(t) \hat{v}_{q c}(t) d t=0$ if $p \neq q$. The above estimation can be implemented by discretizing the covariance estimate $\hat{\Gamma}_{c}(s, t)$ (Rice and Silverman, 1991). The functional principal component score $\xi_{i q c}$ can then be estimated by

$$
\hat{\xi}_{i q c}=\int_{T}\left\{y_{i}(t)-\mathbf{X}_{i}(t)^{T} \hat{\boldsymbol{\beta}}_{c}(t)\right\} \hat{v}_{q c}(t) d t
$$

Let

$$
\hat{y}_{c}\left(t_{i j}\right)=y_{i j}-\sum_{q} \hat{\xi}_{i q c} I\left(\hat{\lambda}_{q c}>0\right) \hat{v}_{q c}\left(t_{i j}\right)
$$

Then, conditioning on $\mathcal{C}=c$, model (2.3) can be approximated by

$$
\hat{y}_{c}\left(t_{i j}\right) \approx \mathbf{X}_{i}\left(t_{i j}\right)^{T} \boldsymbol{\beta}_{c}\left(t_{i j}\right)+e_{i j}
$$

where $e_{i j}$ 's are independent and identically distributed as $N\left(0, \sigma^{2}\right)$. Hence, using functional PCA, we can transform the correlated data to the uncorrelated one. Based on $\left\{\hat{y}_{c}\left(t_{i j}\right), i=1, \ldots, n, j=1, \ldots, N_{i}, c=1, \ldots, C\right\}$ and (2.15), Estimation procedure 1 can be applied to further improve the estimates of $\pi_{c}, \boldsymbol{\beta}_{c}(\cdot)$, and $\sigma_{c}(\cdot)$.

Based on the above discussion, we propose the following improved estimation procedure to incorporate the covariance functions to the model estimation.

\section{Estimation procedure 2 (General Covariance Structure)}

1. Calculate $\hat{\boldsymbol{\beta}}_{c}(\cdot), \hat{\pi}_{c}$, and $\hat{r}_{i c}$ using Estimation procedure 1, and obtain $\hat{y}_{c}\left(t_{i j}\right)$ via $(2.11)-(2.14)$. Let

$$
\hat{\sigma}^{2}=\frac{1}{\sum_{i=1}^{n} N_{i}} \sum_{i=1}^{n} \sum_{c=1}^{C} \sum_{j=1}^{N_{i}} \hat{r}_{i c}\left\{\hat{y}_{c}\left(t_{i j}\right)-\mathbf{X}_{i}\left(t_{i j}\right)^{T} \hat{\boldsymbol{\beta}}_{c}\left(t_{i j}\right)\right\}^{2} .
$$


Then set the initial values $\boldsymbol{\beta}_{c}^{(1)}(\cdot)=\hat{\boldsymbol{\beta}}_{c}(\cdot), \pi_{c}^{(1)}=\hat{\pi}_{c}, r_{i c}^{(1)}=\hat{r}_{i c}, c=1, \ldots, C$, and $\sigma^{2(1)}=\hat{\sigma}^{2}$.

2. Estimate the covariance function $\Gamma_{c}(s, t)$ by

$$
\Gamma_{c}^{(l+1)}(s, t)=\frac{\sum_{i=1}^{n} r_{i c}^{(l)} \sum_{1 \leq j \neq l \leq N_{i}} \gamma_{i c}^{(l)}\left(t_{i j}, t_{i l}\right) K_{h_{\Gamma}}\left(t_{i j}-s\right) K_{h_{\Gamma}}\left(t_{i l}-t\right)}{\sum_{i=1}^{n} r_{i c}^{(l)} \sum_{1 \leq j \neq l \leq N_{i}} K_{h_{\Gamma}}\left(t_{i j}-s\right) K_{h_{\Gamma}}\left(t_{i l}-t\right)},
$$

where $\gamma_{i c}^{(l)}\left(t_{i j}, t_{i l}\right)=\left\{y_{i j}-\mathbf{X}_{i}\left(t_{i j}\right)^{T} \boldsymbol{\beta}_{c}^{(l)}\left(t_{i j}\right)\right\}\left\{y_{i l}-\mathbf{X}_{i}\left(t_{i l}\right)^{T} \boldsymbol{\beta}_{c}^{(l)}\left(t_{i l}\right)\right\}$. Let $\lambda_{q c}^{(l+1)}$ and $v_{q c}^{(l+1)}(\cdot)$ be the estimated eigenvalues and eigenfunctions, respectively, from the covariance estimate $\Gamma_{c}^{(l+1)}(s, t)$. To ensure positive-definiteness of the covariance estimates, we set $\Gamma_{c}^{(l+1)}(s, t)=\sum_{q} \lambda_{q c}^{(l+1)} I\left(\lambda_{q c}^{(l+1)}>0\right) v_{q c}^{(l+1)}(s) v_{q c}^{(l+1)}(t)$.

3. Calculate the transformed response

$$
y_{c}^{(l+1)}\left(t_{i j}\right)=y_{i j}-\sum_{q} \xi_{i q c}^{(l+1)} I\left(\lambda_{q c}^{(l+1)}>0\right) v_{q c}^{(l+1)}\left(t_{i j}\right),
$$

where

$$
\xi_{i q c}^{(l+1)}=\int_{T}\left\{y_{i}(t)-\mathbf{X}_{i}(t)^{T} \boldsymbol{\beta}_{c}^{(l)}(t)\right\} v_{q c}^{(l+1)}(t) d t .
$$

4. One cycle E-step:

$$
r_{i c}^{(l+1)}=\frac{\pi_{c}^{(l)}\left[\prod_{j=1}^{N_{i}} \phi\left\{y_{c}^{(l+1)}\left(t_{i j}\right) \mid \mathbf{X}_{i}\left(t_{i j}\right)^{T} \boldsymbol{\beta}_{c}^{(l)}\left(t_{i j}\right), \sigma^{2(l)}\right\}\right]}{\sum_{c=1}^{C} \pi_{c}^{(l)}\left[\prod_{j=1}^{N_{i}} \phi\left\{y_{c}^{(l+1)}\left(t_{i j}\right) \mid \mathbf{X}_{i}\left(t_{i j}\right)^{T} \boldsymbol{\beta}_{c}^{(l)}\left(t_{i j}\right), \sigma^{2(l)}\right\}\right]} .
$$


5. One cycle M-step: For $t_{0} \in\left\{u_{1}, \cdots, u_{n_{\text {grid }}}\right\}$,

$$
\begin{aligned}
\pi_{c}^{(l+1)} & =\frac{1}{n} \sum_{i=1}^{n} r_{i c}^{(l+1)}, \\
\boldsymbol{\beta}_{c}^{(l+1)}\left(t_{0}\right) & =\left\{\sum_{i=1}^{n} \mathbf{X}_{i}^{T} W_{i c}^{(l+1)}\left(t_{0}\right) \mathbf{X}_{i}\right\}^{-1}\left\{\sum_{i=1}^{n} \mathbf{X}_{i}^{T} W_{i c}^{(l+1)}\left(t_{0}\right) \mathbf{y}_{i c}^{(l+1)}\right\} \\
\sigma^{2(l+1)} & =\frac{1}{\sum_{i=1}^{n} N_{i}} \sum_{i=1}^{n} \sum_{c=1}^{C} \sum_{j=1}^{N_{i}} r_{i c}^{(l+1)}\left\{y_{c}^{(l+1)}\left(t_{i j}\right)-\mathbf{X}_{i}\left(t_{i j}\right)^{T} \boldsymbol{\beta}_{c}^{(l+1)}\left(t_{i j}\right)\right\}^{2},
\end{aligned}
$$

where

$$
\begin{aligned}
\mathbf{y}_{i c}^{(l+1)} & =\left\{y_{c}^{(l+1)}\left(t_{i 1}\right), \cdots, y_{c}^{(l+1)}\left(t_{i N_{i}}\right)\right\}^{T}, \\
W_{i c}^{(l+1)}\left(t_{0}\right) & =\operatorname{diag}\left\{r_{i c}^{(l+1)} K_{h_{\beta}}\left(t_{i 1}-t_{0}\right), \cdots, r_{i c}^{(l+1)} K_{h_{\beta}}\left(t_{i N_{i}}-t_{0}\right)\right\} .
\end{aligned}
$$

6. Iterate Steps $2-5$ with $l=2,3, \ldots$, until convergence.

\section{Model Selection and Inference}

\subsection{Model Selection}

The model selection includes selection of the model type (the full model (2.3) and the reduced model (2.4)), the number of components $C$, the bandwidths $h_{\beta}$ and $h_{\Gamma}$, and the number of eigenfunctions. We discuss these aspects in this section.

Selection of the number of components $C$ is a difficult yet important issue for mixture models. Many efforts have been made to solve this problem for parametric mixture models (Hartigan, 1985; Chen et al., 2001; Li and Chen, 2010; Leroux, 1992; Frühwirth-Schnatter, 2006; McLachlan and Peel, 2000). Bayesian information criterion (BIC) is one of the most extensively used methods. The BIC has the form $-2 \mathcal{L}+\log (n) \times d f$, where $\mathcal{L}$ is the maximum log-likelihood function, and $d f$ is the model degree of freedom which measures the complexity of the model. However, it is not clear how to define the model complexity for the mixture of FLMs, since it contains one-dimensional mean functions smoothing, and the two-dimensional covari- 
ance functions smoothing. Next, we propose to adopt the idea of Fan et al. (2001) to define the effective degree of freedom for nonparametric kernel smoothing and then apply the traditional information criteria to do model selection.

For the one-dimensional mean functions smoothing, based on Fan et al. (2001), we define the effective degree of freedom by

$$
d f_{\beta}=\tau_{K} h_{\beta}^{-1}|\Omega|\left\{K(0)-\frac{1}{2} \int K^{2}(t) d t\right\},
$$

where $\Omega$ is the support of the time $t$, and

$$
\tau_{K}=\frac{K(0)-\frac{1}{2} \int K^{2}(t) d t}{\int\left\{K(t)-\frac{1}{2} K * K(t)\right\}^{2} d t} .
$$

Note that the $d f_{\beta}$ depends on $h_{\beta}^{-1}|\Omega|$. As discussed in Remark 3.2 in Fan et al. (2001), the number of parameters is $h_{\beta}^{-1}|\Omega|$ if the one dimensional support is partitioned into intervals of length $h_{\beta}$, and piecewise constant functions are used for approximation. Similarly, for the two-dimensional covariance functions smoothing, we can define the effective degree of freedom as

$$
d f_{\Gamma}=\tau_{K}^{2} h_{\Gamma}^{-2}|\Omega|^{2}\left\{K(0)-\frac{1}{2} \int K^{2}(t) d t\right\}^{2} .
$$

Based on the above definitions, the degree of freedom for the model $(2.4)$ is $(p C+C) \times$ $d f_{\beta}+C-1$, and the degree of freedom for the model (2.3) is $p \times C \times d f_{\beta}+C \times d f_{\Gamma}+C$. We choose the model with the minimum BIC in the candidate set which consists of the model (2.3) and the model (2.4) with different $C$. Note that the degree of freedom depends on both $C$ and bandwidths. In practice, we can apply BIC for a wide range of bandwidths.

Once the number of components $C$ is determined, we need to choose the bandwidths and the number of eigenfunctions. Choosing the bandwidths has long been a difficult problem for nonparametric and semiparametric models. For a comprehensive review on the bandwidth selection in the kernel regression, see, for example, Marron (1988) and Fan and Gijbels (1996). In this paper, we consider a multifold 
cross-validation (CV) method to choose the bandwidths. In the model (2.4), a conventional CV is applied as we use the same bandwidth for both the regression functions and covariance functions for the simplicity. For the model (2.3), we need to select both $h_{\beta}$ and $h_{\Gamma}$. Hence, CV needs to be performed in a two-dimensional domain. The simulation results in Section 4 demonstrate that the proposed estimation procedure works well for a wide range of bandwidths.

Given selected bandwidths in the model (2.3), the number of eigenfunctions might be chosen using one-curve-leave-out CV and pseudo-AIC criterion (Rice and Silverman, 1991; Yao et al., 2005). From our simulation experience, these methods do not work very well in our model setting. Following Huang et al. (2014), we choose the number of eigenfunctions by an empirical criterion such that the percentage of total variation explained is above certain percentage, such as $95 \%$.

\subsection{Model Inference}

For the proposed model, it is of interest to test whether the coefficient functions $\boldsymbol{\beta}_{c}(t)$ s actually depend on $t$. This leads to the following hypothesis testing problem:

$$
H_{0}: \boldsymbol{\beta}_{c}(t) \equiv \boldsymbol{\beta}_{c}, \quad c=1, \ldots, C
$$

Let $\ell\left(H_{0}\right)$ and $\ell\left(H_{1}\right)$ be the maximum log-likelihoods under null and alternative hypotheses, respectively. Then we construct a likelihood ratio test statistic

$$
T=2\left\{\ell\left(H_{1}\right)-\ell\left(H_{0}\right)\right\}
$$

Since both the null and alternative models are semiparametric ones, such hypothesis test belongs to the Wilks phenomenon and generalized likelihood ratio theory (Fan et al., 2001) for the semiparametric modeling. In this paper, we shall first demonstrate that the Wilk's type of results hold for our model via a Monte Carlo simulation study, i.e., the null distribution of (3.1) does not depend on the nuisance parameters of the null model. Then we apply a conditional bootstrap method (Cai et al., 2000; Fan et al., 1999) to estimate the null distribution. See Section 4 for more detail about its 
implementation. The conditional bootstrap method will also be used for the standard error estimation and to construct pointwise confidence intervals (CIs) for coefficient functions.

\section{Simulation and Application}

To measure the performance of our estimators, we use the root of the average squared errors (RASE). For the estimate of the parameter $\pi$, define $\operatorname{RASE}_{\pi}^{2}=$ $\sum_{c=1}^{C-1}\left\{\hat{\pi}_{c}-\pi_{c}\right\}^{2}$. For the estimates of the regression coefficient functions $\boldsymbol{\beta}_{c}(t)$, define

$$
\operatorname{RASE}_{\beta}^{2}=n_{\text {grid }}^{-1} \sum_{c=1}^{C} \sum_{j=1}^{n_{\text {grid }}}\left\|\hat{\boldsymbol{\beta}}_{c}\left(u_{j}\right)-\boldsymbol{\beta}_{c}\left(u_{j}\right)\right\|^{2},
$$

where $\left\{u_{j}, j=1, \cdots, n_{\text {grid }}\right\}$ are a set of grid points. The number of grid points is set to be $n_{\text {grid }}=50$, and all the grid points are evenly distributed on the range of $t$.

\subsection{Simulation Study}

We shall consider two simulation scenarios as follow.

\section{Scenario 1}

$$
\begin{aligned}
& \pi_{1}=0.6, \quad \pi_{2}=1-\pi_{1}=0.4, \text { and } \sigma^{2}=0.25, \\
& \boldsymbol{\beta}_{1}(t)=(\sin (\pi t), \cos (2 \pi t)) \text { and } \boldsymbol{\beta}_{2}(t)=\left(t^{2}-3, \sin (2 \pi t)+3\right), \\
& v_{11}(t)=\sqrt{2} \sin (4 \pi t) \text { and } v_{21}(t)=\sqrt{2} \cos (4 \pi t), \\
& v_{12}(t)=\sqrt{2} \sin (\pi t) \text { and } v_{22}(t)=\sqrt{2} \cos (\pi t), \\
& \lambda_{1 c}=0.04, \quad \lambda_{2 c}=0.01 \text { and } \lambda_{q c}=0, \text { for } q>2, \quad c=1,2 .
\end{aligned}
$$




\section{Scenario 2}

$$
\begin{aligned}
& \pi_{1}=0.45, \quad \pi_{2}=1-\pi_{1}=0.55, \text { and } \sigma^{2}=0.25, \\
& \boldsymbol{\beta}_{1}(t)=(0, \sin (\pi t)) \text { and } \boldsymbol{\beta}_{2}(t)=(0,1.5 \sin (\pi t)), \\
& v_{11}(t)=\sqrt{2} \sin (4 \pi t) \text { and } v_{21}(t)=\sqrt{2} \cos (4 \pi t), \\
& v_{12}(t)=\sqrt{2} \sin (\pi t) \text { and } v_{22}(t)=\sqrt{2} \cos (\pi t), \\
& \lambda_{11}=0.16, \quad \lambda_{21}=0.04 \text { and } \lambda_{q 1}=0, \text { for } q>2, \\
& \lambda_{12}=0.04, \quad \lambda_{22}=0.01 \text { and } \lambda_{q 2}=0, \text { for } q>2 .
\end{aligned}
$$

In the first scenario, the two components are well-separated; while in the second scenario, the two components heavily overlap. In both scenarios, the two components have different correlation structures. For each scenario, the simulated data of size $n=100$ are observed at grid points $\{k / N, k=1, \cdots, N\}$ for both components, where $N$ is set to be 20 and 40, respectively. At each grid point, the predictor $X$ is generated from a one-dimensional standard normal distribution. The principal component scores $\xi_{i q c}$ are generated from $N\left(0, \lambda_{q c}\right), q=1,2$, and $c=1,2$. Typical samples of the response from the two scenarios are depicted in Figure 2.

Notations $M_{F}$ and $M_{R}$ stand for the full model (2.3) with the estimation procedure 2, and the reduced model (2.4) with the estimation procedure 1, respectively. The Epanechnikov kernel is used for the kernel smoothing in estimation.

We first test the performance of the proposed model selection method based on BIC and the defined effective degree of freedom for BIC. To illustrate the method, we design a contrast scenario $1 b$ which is the same as scenario 1 except that it has an independent covariance structure. 


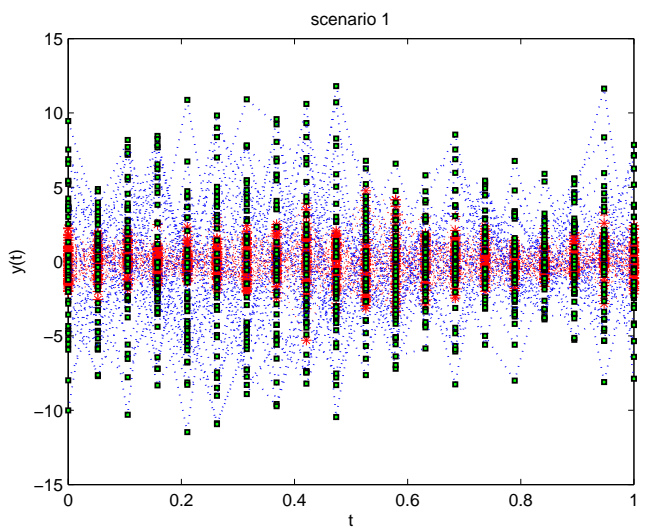

(a)

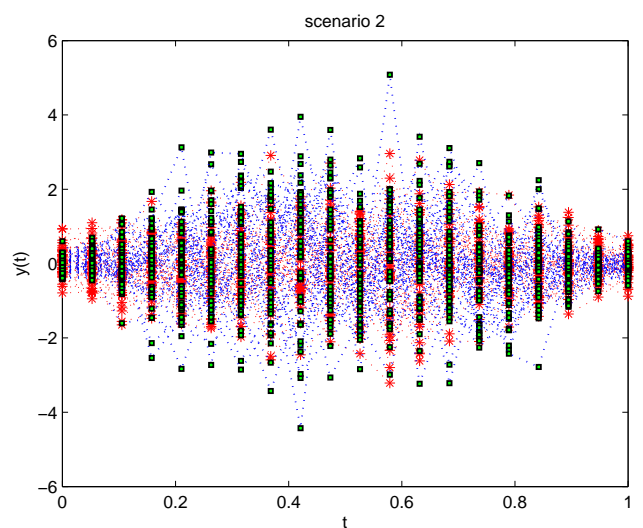

(b)

Figure 2: (a) Response of a typical sample for the well-separated setting; (b) Response of typical sample for heavy-overlap setting.

\section{Scenario $1 b$}

$$
\begin{aligned}
& \pi_{1}=0.6, \quad \pi_{2}=1-\pi_{1}=0.4 \\
& \boldsymbol{\beta}_{1}(t)=(\sin (\pi t), \cos (2 \pi t)) \text { and } \boldsymbol{\beta}_{2}(t)=\left(t^{2}-3, \sin (2 \pi t)+3\right), \\
& \sigma_{1}^{2}(t)=0.2 \sin (\pi t)+0.25 \text { and } \sigma_{2}^{2}(t)=0.3 \sin (\pi t)+0.25
\end{aligned}
$$

For both scenarios 1 and 1b, we fit the mixture of FLMs under both the full model $\left(M_{F}\right)$ and the reduced model $\left(M_{R}\right)$ with 1,2 , and 3 components under 9 different pairs of bandwidths where $h_{\beta} \in\{0.06,0.08,0.10\}$ and $h_{\Gamma} \in\{0.28,0.35,0.42\}$, and then compare the corresponding BIC scores. The $C$ is selected by minimizing the BIC scores over $M_{F}$ and $M_{R}$, the three values of $C$, and the 9 pairs of bandwidths $\left(h_{\beta}, h_{\Gamma}\right)$. The frequencies of selected $C$ s in 100 simulations are presented in Table 1. From these results, we can see that for the data with independent covariance structure (scenario $1 \mathrm{~b}$ ), the proportions for BIC to choose the correct $C$ and the reduced model (2.4) are 100\%; while for the data with non-isotropic covariance structure (scenario 1 ), the proportion of selecting the correct $C$ is $100 \%$, and the proportion of selecting the correct $C$ and the full model $(2.3)$ is $94 \%$. The above results demonstrate the effectiveness of the proposed model selection method. In addition, the new method 
Table 1: Frequencies of the selected $C$ and the model type by BIC $(N=20)$

\begin{tabular}{cccc}
\hline & & Scenario 1 & Scenario 1b \\
\hline \multirow{4}{*}{$M_{R}$} & $\mathrm{C}=1$ & 0 & 0 \\
& $\mathrm{C}=2$ & 6 & 100 \\
& $\mathrm{C}=3$ & 0 & 0 \\
\hline \multirow{3}{*}{$M_{F}$} & $\mathrm{C}=1$ & 0 & 0 \\
& $\mathrm{C}=2$ & 94 & 0 \\
& $\mathrm{C}=3$ & 0 & 0 \\
\hline
\end{tabular}

can not only choose the correct number of components, but also identify the proper model $\left(M_{F}\right.$ or $\left.M_{R}\right)$ to use.

In the following simulation, we assume that the number of components $C$ is known. For each simulated dataset, we obtain the optimal bandwidths for coefficient and covariance functions using a 5-fold CV method. However, such a CV method performs well at a computational expense. To ease the computation burden, we fix the bandwidth pairs for each simulated dataset. The bandwidth pairs in Table 2 are selected as the average of optimal CV bandwidths of several simulated datasets. From the table, we can see that both $h_{\beta}$ and $h_{\Gamma}$ decrease as $N$ increases. Table 3 reports the mean and standard deviation of $\mathrm{RASE}_{\beta}, \mathrm{RASE}_{\pi}$, and the estimated $\pi$ for both scenarios 1 and 2 over 500 simulations. The results show that $M_{R}$ and $M_{F}$ have similar performance and work well with the selected bandwidths in scenario 1 . In contrast, in scenario $2, M_{F}$ does a much better job than $M_{R}$, producing less bias for the parameter $\pi_{1}$, and smaller RASE $\beta$ and $\mathrm{RASE}_{\pi}$. Therefore, when the mixture components are close, incorporating the covariance functions into the model estimation using $M_{F}$ could significantly improve the accuracy of the model estimation.

Next, we conduct a simulation study to investigate the Wilk's type of phenomenon for the hypothesis testing of coefficient functions. The simulated data are generated according to scenario 2, with the only difference that the coefficient functions are constants. We take three different values of the constant $\boldsymbol{\beta}_{c},\{(0,-0.5),(0,0.5)\}$, $\{(0,-0.5),(0,1)\}$, and $\{(0,0.5),(0,1.5)\}$. Since the covariances are non-isotropic, we 
Table 2: Optimal bandwidth pairs $\left(\hat{h}_{\beta}, \hat{h}_{\Gamma}\right)$

\begin{tabular}{cccc}
\hline & & $M_{R}$ & $M_{F}$ \\
\hline Scen. & $N$ & $h_{\beta}$ & $h_{\Gamma}$ \\
\hline 1 & 20 & 0.0805 & 0.3500 \\
& 40 & 0.0730 & 0.1290 \\
\hline 2 & 20 & 0.0650 & 0.1620 \\
& 40 & 0.0560 & 0.0800 \\
\hline
\end{tabular}

Table 3: Mean and standard deviation of RASEs

\begin{tabular}{cccccccc}
\hline \multicolumn{7}{c}{$M_{R}$} & \multicolumn{3}{c}{$M_{F}$} \\
\hline Scen. & $N$ & $\mathrm{RASE}_{\beta}$ & $\mathrm{RASE}_{\pi}$ & $\pi_{1}=0.6$ & $\mathrm{RASE}_{\beta}$ & $\mathrm{RASE}_{\pi}$ & $\pi_{1}=0.6$ \\
\hline 1 & 20 & $0.013(0.003)$ & $0.002(0.003)$ & $0.602(0.050)$ & $0.013(0.003)$ & $0.002(0.003)$ & $0.602(0.050)$ \\
& 40 & $0.008(0.002)$ & $0.002(0.003)$ & $0.601(0.047)$ & $0.007(0.001)$ & $0.002(0.003)$ & $0.601(0.047)$ \\
\hline Scen. & $N$ & $\mathrm{RASE}_{\beta}$ & $\mathrm{RASE}_{\pi}$ & $\pi_{1}=0.45$ & $\mathrm{RASE}_{\beta}$ & $\mathrm{RASE}_{\pi}$ & $\pi_{1}=0.45$ \\
\hline 2 & 20 & $0.059(0.288)$ & $0.024(0.071)$ & $0.489(0.149)$ & $0.009(0.043)$ & $0.003(0.014)$ & $0.454(0.057)$ \\
& 40 & $0.017(0.063)$ & $0.021(0.068)$ & $0.496(0.139)$ & $0.002(0.014)$ & $0.003(0.004)$ & $0.451(0.051)$ \\
\hline
\end{tabular}

incorporate the covariance functions in the estimation for both models under the null and alternative hypotheses, and the corresponding log-likelihoods $\ell\left(H_{0}\right)$ and $\ell\left(H_{1}\right)$ are calculated. Let $T$ denote the likelihood ratio test statistic. We compute the unconditional null distributions of $T$ based on 500 Monte Carlo simulations. The resulting three density estimates are very close to each other, plotted as dotted lines in Figure 3 (a). As expected, the asymptotic distribution of $T$ under the null hypothesis is not sensitive to the true values of $\boldsymbol{\beta}_{c}$. Next, to validate the conditional bootstrap method, we choose three typical samples generated using these three different $\boldsymbol{\beta}_{c}$. For each typical sample, we first obtain the estimates, and then compute the conditional null distributions of $T$ based on 500 parametric bootstrap samples. The resulting three densities are depicted as solid curves in Figure 3 (b). From Figure 3 (b), we can see that the conditional bootstrapped distributions are very close and work reasonably well in approximating the true null distribution.

Next, we investigate the accuracy of the conditional bootstrap method to estimate 


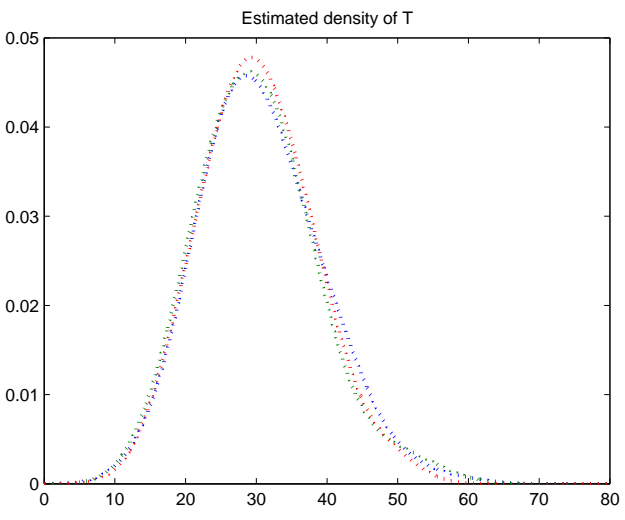

(a)

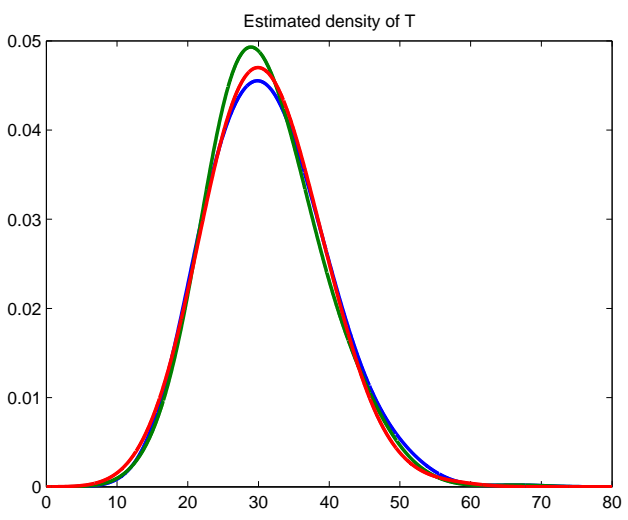

(b)

Figure 3: (a) The estimated densities of unconditional null distributions of $T$ for three different null hypotheses; (b) the estimated densities of conditional null distributions of $T$ (solid lines). The online version of this figure is in color.

Table 4: Standard errors via bootstrap (scenario $1, N=20$ )

\begin{tabular}{ccccccccccc}
\hline & & 0.1 & 0.2 & 0.3 & 0.4 & 0.5 & 0.6 & 0.7 & 0.8 & 0.9 \\
\hline \multirow{3}{*}{$\beta_{11}(\cdot)$} & SD & 0.044 & 0.044 & 0.049 & 0.050 & 0.047 & 0.044 & 0.048 & 0.047 & 0.040 \\
& SE & 0.042 & 0.043 & 0.044 & 0.045 & 0.046 & 0.046 & 0.044 & 0.043 & 0.042 \\
& Std & 0.003 & 0.004 & 0.004 & 0.004 & 0.005 & 0.005 & 0.004 & 0.004 & 0.004 \\
\hline \multirow{3}{*}{$\beta_{12}(\cdot)$} & SD & 0.043 & 0.051 & 0.054 & 0.054 & 0.051 & 0.047 & 0.052 & 0.050 & 0.042 \\
& SE & 0.042 & 0.044 & 0.046 & 0.046 & 0.046 & 0.046 & 0.045 & 0.043 & 0.043 \\
& Std & 0.004 & 0.004 & 0.005 & 0.005 & 0.004 & 0.005 & 0.004 & 0.004 & 0.004 \\
\hline \multirow{2}{*}{$\beta_{21}(\cdot)$} & SD & 0.052 & 0.056 & 0.044 & 0.057 & 0.058 & 0.063 & 0.054 & 0.050 & 0.056 \\
& SE & 0.053 & 0.052 & 0.052 & 0.055 & 0.059 & 0.056 & 0.054 & 0.052 & 0.053 \\
& Std & 0.006 & 0.006 & 0.005 & 0.006 & 0.007 & 0.006 & 0.005 & 0.005 & 0.005 \\
\hline \multirow{3}{*}{$\beta_{22}(\cdot)$} & SD & 0.054 & 0.054 & 0.054 & 0.061 & 0.062 & 0.062 & 0.050 & 0.057 & 0.057 \\
& SE & 0.054 & 0.051 & 0.053 & 0.058 & 0.061 & 0.057 & 0.052 & 0.052 & 0.054 \\
& Std & 0.006 & 0.006 & 0.006 & 0.006 & 0.007 & 0.006 & 0.006 & 0.005 & 0.005 \\
\hline
\end{tabular}

Table 5: Standard errors via bootstrap (scenario 2, $N=20$ )

\begin{tabular}{ccccccccccc}
\hline & & 0.1 & 0.2 & 0.3 & 0.4 & 0.5 & 0.6 & 0.7 & 0.8 & 0.9 \\
\hline \multirow{3}{*}{$\beta_{12}(\cdot)$} & SD & 0.021 & 0.024 & 0.021 & 0.023 & 0.025 & 0.024 & 0.025 & 0.023 & 0.026 \\
& SE & 0.025 & 0.026 & 0.026 & 0.027 & 0.025 & 0.027 & 0.027 & 0.027 & 0.025 \\
& Std & 0.003 & 0.003 & 0.003 & 0.003 & 0.003 & 0.003 & 0.003 & 0.003 & 0.003 \\
\hline \multirow{3}{*}{$\beta_{22}(\cdot)$} & SD & 0.026 & 0.025 & 0.020 & 0.022 & 0.019 & 0.019 & 0.020 & 0.023 & 0.024 \\
& SE & 0.023 & 0.024 & 0.023 & 0.022 & 0.022 & 0.022 & 0.023 & 0.024 & 0.023 \\
& Std & 0.003 & 0.003 & 0.003 & 0.003 & 0.002 & 0.003 & 0.003 & 0.003 & 0.003 \\
\hline
\end{tabular}


the standard errors for the coefficient estimates. We use SE and Std to denote the average and the standard deviation of 200 bootstrapped standard errors, respectively, and use SD to denote the standard deviation of the 200 estimates. SD can be considered as the "real" standard errors, and serves as a benchmark for the comparison. These quantities are given in Tables 4 and 5. From the two tables, we can see that SD and SE are very close, which demonstrates the effectiveness of the conditional bootstrap method as a tool to provide the standard error estimates.

Now we compare our model with an existing alternative procedure, the the random effects regression mixture (RERM) model (DeSarbo and Cron, 1988; Verbeke and Lesaffre, 1996; Xu and Hedeker, 2001), with a spline representation for the nonparametric structure. Conditioning on the component membership $\mathcal{C}=c$,

$$
y_{i}(t)=\mathbf{X}_{i}(t)^{T} \boldsymbol{\beta}_{c}(t)+e_{i}(t)
$$

where $e(t)$ is an uncorrelated process with a constant variance function $\sigma^{2}(t) \equiv \sigma^{2}$. We assume that element functions in $\boldsymbol{\beta}_{c}(t)=\left\{\beta_{c 1}(t), \cdots, \beta_{c p}(t)\right\}$ are approximated by the following sum of the spline basis $B_{k}(t)$ :

$$
\beta_{c j}(t)=\sum_{k=1}^{K} \gamma_{c j k} B_{k}(t),
$$

for $j=1, \cdots, p$. Let $\gamma_{c j}=\left(\gamma_{c j 1}, \ldots, \gamma_{c j K}\right)^{T}$. For the random effects regression mixture (RERM) model with a spline representation, we assume that $\boldsymbol{\gamma}_{c j} \sim N\left(\boldsymbol{\mu}_{c j}, \mathbf{R}_{c j}\right)$. The unknown parameters in the model are $\left\{\pi_{c}, \boldsymbol{\mu}_{c j}, \mathbf{R}_{c j}\right\}$ for $c=1, \cdots, C ; j=$ $1, \cdots, p$, under constrains $\pi_{c}>0$, and $\sum_{c=1}^{C} \pi_{c}=1$. The parameters can be estimated using standard MLE procedure (e.g., EM algorithm) of RERM. Let $\left\{\hat{\pi}_{c}, \hat{\boldsymbol{\mu}}_{c j}, \hat{\mathbf{R}}_{c j}\right\}$ be the estimates. Then the mean function estimation can be expressed as

$$
\hat{\beta}_{c j}(t)=\sum_{k=1}^{K} \hat{\mu}_{c j k} B_{k}(t),
$$

where $\hat{\mu}_{c j k}$ are elements of $\boldsymbol{\mu}_{c j}$. For convenience, we use a cubic-spline representation 
Table 6: Mean and Standard Deviation of RASEs

\begin{tabular}{|c|c|c|c|c|c|c|c|}
\hline & & \multicolumn{3}{|c|}{ RERM } & \multicolumn{3}{|c|}{$M_{F}$} \\
\hline Scen. & $N$ & $\operatorname{RASE}_{\beta}$ & $\mathrm{RASE}_{\pi}$ & $\pi_{1}=0.6$ & $\operatorname{RASE}_{\beta}$ & $\mathrm{RASE}_{\pi}$ & $\pi_{1}=0.6$ \\
\hline \multirow[t]{2}{*}{1} & 20 & $0.005(0.002)$ & $0.003(0.004)$ & $0.597(0.050)$ & $0.013(0.003)$ & $0.002(0.003)$ & $0.600(0.048)$ \\
\hline & 40 & $0.003(0.001)$ & $0.003(0.004)$ & $0.599(0.050)$ & $0.007(0.001)$ & $0.003(0.004)$ & $0.606(0.051)$ \\
\hline Scen. & $N$ & $\mathrm{RASE}_{\beta}$ & $\mathrm{RASE}_{\pi}$ & $\pi_{1}=0.45$ & $\operatorname{RASE}_{\beta}$ & $\mathrm{RASE}_{\pi}$ & $\pi_{1}=0.45$ \\
\hline \multirow[t]{2}{*}{2} & 20 & $0.011(0.036)$ & $0.012(0.023)$ & $0.385(0.091)$ & $0.009(0.043)$ & $0.003(0.014)$ & $0.454(0.057)$ \\
\hline & 40 & $0.003(0.016)$ & $0.004(0.018)$ & $0.438(0.065)$ & $0.002(0.014)$ & $0.003(0.004)$ & $0.451(0.051)$ \\
\hline
\end{tabular}

with evenly spaced knots. The number of knots is chosen such that the degree of freedom of the RERM is about the same as the effective degree of freedom of our proposed model (see section 3.1 of our paper). We perform the calculation using $\mathrm{R}$ package "mixtools" (see Young et al., 2007).

We summarize the comparison results in Table 6. Notations "RERM" and " $M_{F}$ " stand for the random effects regression mixture model and our model respectively. The two scenarios are the same as those in section 4.1. From Table 6, we can see that when the two components are well-separated (Scenario 1), both estimation procedures give similar results for the estimation of $\pi$, while RERM has better estimation of $\boldsymbol{\beta}(t)$. When the components are heavily overlap (Scenario 2), the proposed procedure $M_{F}$ gives better results compared to RERM.

We also compare our method with the fully nonparametric functional regression model (NFRM) of Ferraty et al. (2012). We use the scenario $2(N=20)$ as an illustration. Each dataset is split randomly into a training sample of 75 observations and a testing sample of 25 observations, and the process is replicated 100 times. The training sample is used to select and estimate the parameters of the models and the testing sample is used to compare prediction performance for the two models. The criteria for prediction comparison are the absolute error $A E=\Sigma_{i}\left|Y_{i}-\hat{Y}_{i}\right|$ and the quadratic error $Q E=\Sigma_{i}\left(Y_{i}-\hat{Y}_{i}\right)^{2}$. Table 7 gives the mean values of $A E$ and $Q E$ and shows that our model works better in terms of the prediction errors. 
Table 7: Mean value of the criterion errors on the test sample

\begin{tabular}{ccc}
\hline Model & Absolute error & Quadratic error \\
\hline NFRM & 13.050 & 15.574 \\
$M_{F}$ & 3.388 & 1.187 \\
\hline
\end{tabular}

\section{2. $\mathrm{CO}_{2}-G D P$ data application}

In this section, we analyze the $\mathrm{CO}_{2}$-GDP data using the proposed model and estimation procedure. The data record the $\mathrm{CO}_{2}$ emission per capita and the GDP per capita for 175 countries from 1980 to 2005. It has a balanced structure, with one observation each year for each country. The trajectories of the $\mathrm{CO}_{2}$ emission for all the 175 countries are assembled in Figure 1(a). Huang and Yao (2012) showed that a cross-sectional subset of the data in year 2005 can be modeled by a 2-component mixture of regression models with varying proportion, with each component revealing a linear effect of the GDP per capita on the $\mathrm{CO}_{2}$ emission per capita. In this study, we are interested in whether this linear effect of the GDP on the $\mathrm{CO}_{2}$ emission varies over time for the two components.

We first choose the model type and the number of components $C$ via BIC. Using bandwidths $h_{\beta} \in\{0.07,0.09,0.11\}$ and $h_{\Gamma} \in\{0.11,0.13,0.15\}$, we fit a mixture of FLMs by $M_{F}$ and $M_{R}$ to the data with one, two, three, and four components, respectively. Then we calculate and compare their corresponding BIC scores. For these bandwidths, the minimum BIC score is achieved at $C=2$ with $M_{F}$. Hence a twocomponent mixture model is selected. This agrees with the cross-sectional analysis by Huang and Yao (2012). Our result also indicates that we should use the full model (2.3) and the estimation procedure two to incorporate the correlation structure. With $M_{F}$ and $C=2$, a 5 -fold CV suggests that the optimal bandwidths for estimating coefficient and covariance functions are 0.085 and 0.13 , respectively. With these optimal bandwidths, the $95 \%$ percent rule-of-thumb criterion selects 2 eigenfunctions for each component. We then apply the test procedure from Section 3.2 to test whether the 


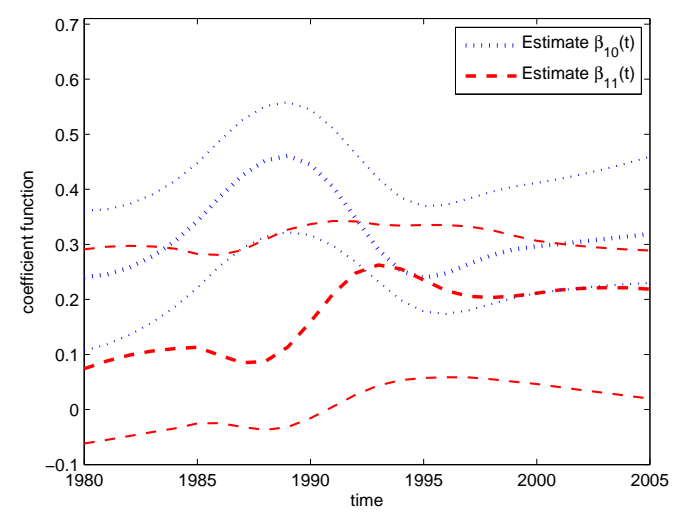

(a)

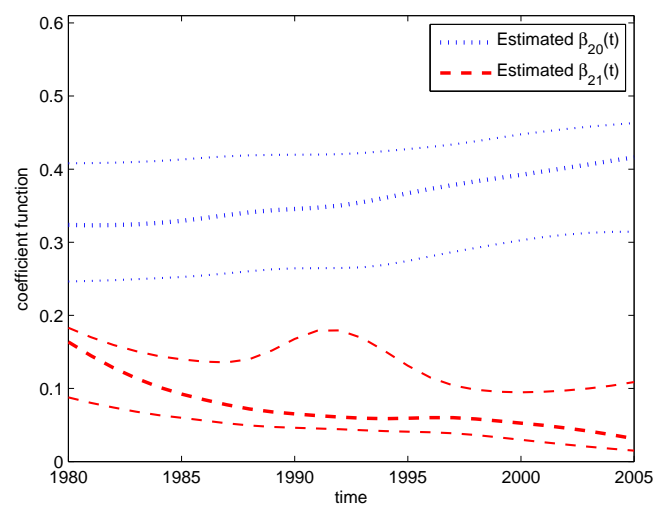

(b)

Figure 4: The estimated coefficient functions with the $95 \%$ pointwise confidence intervals for the $\mathrm{CO}_{2}$-GDP data. (a) Coefficient functions of component 1 ; (b) coefficient functions of component 2.

coefficient functions change over time. The test statistic $T$ is 571.81 using the estimation procedure 2 , with $p$-value $(<0.01)$ being close to zero. Therefore, we reject the null hypotheses of constant coefficient functions, and conclude that a mixture of FLMs with general covariance structure is needed for the analysis of the $\mathrm{CO}_{2}$-GDP data.

The estimated mixing proportions for the two components are 0.1524 and 0.8476 , respectively. We label the component with the proportion 0.1524 as the first component, and the other as the second component. Countries in the first component are those of low GDP per capita and relative high $\mathrm{CO}_{2}$ emissions, including Kuwait, United Arab Emirates, Russian Federation, Georgia, etc. Countries in the second component are those of high GDP per capita with relative low $\mathrm{CO}_{2}$ emissions and thus have healthier economic development path. Representatives in the second component are the United States, Canada, Australia, France, etc. Two estimated coefficient functions $\boldsymbol{\beta}_{c}(t)$ are shown in Figure 4, together with their bootstrapped point-wise confidence intervals. Figure 4(a) demonstrates that the slope function for the first component increases with time for the period of 1980 to 1994, and then remains relatively flat at a high level from 1995 to 2005. In contrast, Figure 4(b) shows 
that for countries in the second component, the slope function decreases slowly over the period of 1980-2005. Therefore, for the countries in component 2 , the increment of the $\mathrm{CO}_{2}$ emissions per capita associated with a unit increment of the GDP per capita decreases slowly over the period of 1980-2005, and is lower than that of the component 1 after 1985. Therefore, the countries in the second component had been improving their economic structure along the years. Our result is in agreement with the finding of Garnaut et al. (2008) on emissions/GDP elasticity in general.

We now use NFRM of Ferraty et al. (2012) to analyze the $\mathrm{CO}_{2}$-GDP data and compare the result with the analysis above. Similar to what we did in simulation, we randomly split the dataset into a training sample of 125 observations and a testing sample of 50 observations, and repeat it 100 times. The training and testing samples are used for parameter estimations and prediction testing respectively. The mean values of $A E$ and $Q E$ are given in Table 8, which demonstrate again the our method performs favorably.

Table 8: Mean value of the criterion errors on the test sample

\begin{tabular}{ccc}
\hline Model & Absolute error & Quadratic error \\
\hline$N F R M$ & 5.375 & 3.831 \\
$M_{F}$ & 0.712 & 0.153 \\
\hline
\end{tabular}

\section{Concluding Remarks}

In this paper, we proposed a new class of mixture of functional linear models to study relationship in inhomogeneous functional data. We showed that the proposed mixture models are identifiable under mild conditions. We developed estimation procedures using the kernel regression, the EM algorithm, and the functional principal component analysis. In order to check whether the coefficient functions are actually varying over time, we employed a semiparametric maximum likelihood ratio test and estimated its null distribution by a conditional bootstrap method. Simulation 
studies and a real data application demonstrated the effectiveness of the proposed methodology.

The simulated and real data in this paper are observed at regular grid points. For irregular and unbalanced data, one may linearly interpolate the data over a regular grid points, and then apply our estimation procedure. Theoretical properties, such as the consistency and the asymptotic normality, of the proposed estimation procedure have not been established. One might be able to establish these properties in the spirit of the work of Hoshikawa (2013). It requires more research.

\section{Acknowledgements}

The authors thank the editor, the associate editor, and reviewers for their constructive comments that have led to a dramatic improvement of the earlier version of this article. Wang's research was partially supported by NSFC grant (11371235) and SRF for ROCS, SEM. Huang's research was partially supported by NSFC grant (11301324). Yao's research was supported by NSF grant DMS-1461677.

\section{Appendix}

Lemma 1. Consider mixtures of linear regression models with Gaussian errors

$$
Y \mid \mathbf{x} \sim \sum_{c=1}^{C} \pi_{c} N\left(\mathbf{x}^{T} \boldsymbol{\beta}_{c}, \sigma_{c}^{2}\right),
$$

where $\boldsymbol{\beta}_{c}=\left(\beta_{0 c}, \beta_{1 c}, \ldots, \beta_{p c}\right)^{T}$. Suppose that $\left(\boldsymbol{\beta}_{c}, \sigma_{c}^{2}\right), c=1, \ldots, C$, are distinct pairs, and that the domain $\mathcal{X}$ of $\mathbf{x}$ contains an open subset in $\mathbb{R}^{p}$. Then the above mixture of linear regression models is identifiable.

Lemma 1 can be viewed as a special case of Theorem 1 from Huang and Yao (2012) with constant proportions. Hennig (2000) also obtained a similar result earlier.

Lemma 2. The multivariate Gaussian mixtures are identifiable. 
This lemma is the Proposition 2 from Yakowitz and Spragins (1968).

Proof of Theorem 1. The proof can be done in a similar manner to Huang et al. (2014). Suppose that $\{Y(t), t \in T\}$ admits another representation such that given a latent variable $\mathcal{G}=g,\{Y(t), t \in T\}$ follows a Gaussian process with mean function $\mathbf{X}(t)^{T} \boldsymbol{\gamma}_{g}(t)$ and covariance function $\operatorname{Cov}\{Y(s), Y(t)\}=\Omega_{g}(s, t), g=1, \ldots, G$. In addition, $P(\mathcal{G}=g)=\lambda_{g}$. Therefore,

$$
Y(t) \sim \sum_{g=1}^{G} \lambda_{g} N\left(\mathbf{X}(t)^{T} \boldsymbol{\gamma}_{g}(t), \Omega_{g}(t, t)\right)=\sum_{c=1}^{C} \pi_{c} N\left(\mathbf{X}(t)^{T} \boldsymbol{\beta}_{c}(t), \Gamma_{c}(t, t)\right) .
$$

For any fixed $t \in T \backslash \mathbf{S},\left(\boldsymbol{\beta}_{c}(t), \Gamma_{c}(t, t)\right), c=1, \ldots, C$, are distinct pairs. By lemma 1, $G=C$, and there exists a permutation $\omega_{t}=\left\{\omega_{t}(1), \ldots, \omega_{t}(C)\right\}$ which may depend on $t$ such that

$$
\lambda_{\omega_{t}(c)}=\pi_{c}, \gamma_{\omega_{t}(c)}(t)=\boldsymbol{\beta}_{c}(t), \Omega_{\omega_{t}(c)}(t, t)=\Gamma_{c}(t, t), c=1, \ldots, C
$$

Now let $s \in T \backslash \mathbf{S}$ and $r \in T$ be two other time points such that $r, s$ and $t$ are all distinct. Then $(Y(r), Y(s), Y(t))^{T}$ follows a mixture of 3-dimensional Gaussian distributions

$$
(Y(r), Y(s), Y(t))^{T} \sim \sum_{c=1}^{C} \lambda_{c} N_{3}\left(\boldsymbol{\nu}_{c}(r, s, t), \boldsymbol{\Omega}_{c}(r, s, t)\right)=\sum_{c=1}^{C} \pi_{c} N_{3}\left(\boldsymbol{\mu}_{c}(r, s, t), \boldsymbol{\Gamma}_{c}(r, s, t)\right) .
$$

where

$$
\begin{array}{cc}
\boldsymbol{\nu}_{c}(r, s, t)=\left(\begin{array}{c}
\mathbf{x}(r)^{T} \boldsymbol{\gamma}_{c}(r) \\
\mathbf{x}(s)^{T} \boldsymbol{\gamma}_{c}(s) \\
\mathbf{x}(t)^{T} \boldsymbol{\gamma}_{c}(t)
\end{array}\right) & \boldsymbol{\Omega}_{c}(r, s, t)=\left(\begin{array}{lll}
\Omega_{c}(r, r) & \Omega_{c}(r, s) & \Omega_{c}(r, t) \\
\Omega_{c}(s, r) & \Omega_{c}(s, s) & \Omega_{c}(s, t) \\
\Omega_{c}(t, r) & \Omega_{c}(t, s) & \Omega_{c}(t, t)
\end{array}\right) \\
\boldsymbol{\mu}_{c}(r, s, t)=\left(\begin{array}{l}
\mathbf{x}(r)^{T} \boldsymbol{\beta}_{c}(r) \\
\mathbf{x}(s)^{T} \boldsymbol{\beta}_{c}(s) \\
\mathbf{x}(t)^{T} \boldsymbol{\beta}_{c}(t)
\end{array}\right) & \boldsymbol{\Gamma}_{c}(r, s, t)=\left(\begin{array}{lll}
\Gamma_{c}(r, r) & \Gamma_{c}(r, s) & \Gamma_{c}(r, t) \\
\Gamma_{c}(s, r) & \Gamma_{c}(s, s) & \Gamma_{c}(s, t) \\
\Gamma_{c}(t, r) & \Gamma_{c}(t, s) & \Gamma_{c}(t, t)
\end{array}\right)
\end{array}
$$

Since $s, t \in T \backslash \mathbf{S}$, from lemma 2, the distribution of $(Y(r), Y(s), Y(t))^{T}$ as a mixture of 3-dimensional Gaussian distributions is identifiable, hence the permutation 
$\omega_{t}$ is actually independent of $t$. Therefore, for any $t_{1}, t_{2} \in\{r, s, t\}$,

$$
\lambda_{\omega(c)}=\pi_{c}, \mathbf{X}\left(t_{1}\right)^{T} \boldsymbol{\gamma}_{\omega(c)}\left(t_{1}\right)=\mathbf{X}\left(t_{1}\right)^{T} \boldsymbol{\beta}_{c}\left(t_{1}\right), \Omega_{\omega(c)}\left(t_{1}, t_{2}\right)=\Gamma_{c}\left(t_{1}, t_{2}\right), c=1, \ldots, C
$$

and for $t_{3} \in\{s, t\}$,

$$
\gamma_{\omega(c)}\left(t_{3}\right)=\boldsymbol{\beta}_{c}\left(t_{3}\right), \quad \Omega_{\omega(c)}\left(t_{3}, t_{3}\right)=\Gamma_{c}\left(t_{3}, t_{3}\right)
$$

By the continuity of $\boldsymbol{\beta}_{c}(\cdot)$ and $\Gamma_{c}(\cdot, \cdot)$, equation (5.4) also holds for $t_{3}=r$. This completes the proof of identifiability.

\section{Derivation of Estimation Procedure 1.}

Define the random variables for component membership as

$$
z_{i c}= \begin{cases}1, & \text { if }\left\{y_{i}(t), t \in T\right\} \text { is in the } c^{t h} \text { group } \\ 0, & \text { otherwise }\end{cases}
$$

The complete likelihood of $\left\{\left(y_{i j}, z_{i c}\right), j=1, \cdots, N_{i}, i=1, \cdots, n, c=1, \ldots C\right\}$ is

$$
\prod_{i=1}^{n} \prod_{c=1}^{C}\left[\pi_{c} \prod_{j=1}^{N_{i}} \phi\left\{y_{i j} \mid X_{i}\left(t_{i j}\right)^{T} \boldsymbol{\beta}_{c}\left(t_{i j}\right), \sigma_{c}^{* 2}\left(t_{i j}\right)\right\}\right]^{z_{i c}} \text {. }
$$

In the E-step, we calculate the expectation of $z_{i c}$ given $\pi_{c}^{(l)}, \sigma_{c}^{* 2(l)}(\cdot)$, and $\boldsymbol{\beta}_{c}^{(l)}(\cdot)$, $c=1, \cdots, C$, which is showed in (2.7). In the M-step, we maximize the logarithm of complete log-likelihood function with $z_{i c}$ replaced by $r_{i c}^{(l+1)}$, which is

$$
\sum_{i=1}^{n} \sum_{c=1}^{C}\left[r_{i c}^{(l+1)} \log \left(\pi_{c}\right)+r_{i c}^{(l+1)} \sum_{j=1}^{N_{i}} \log \phi\left\{y_{i j} \mid X_{i}\left(t_{i j}\right)^{T} \boldsymbol{\beta}_{c}\left(t_{i j}\right), \sigma_{c}^{* 2}\left(t_{i j}\right)\right\}\right] .
$$

The maximization with respect to $\pi_{c}$ leads to (2.8). For nonparametric smoothing functions $\boldsymbol{\beta}_{c}(\cdot)$ and $\sigma_{c}^{* 2}(\cdot)$, we consider kernel regression for estimation. For any $t_{0} \in$ $T$, we approximate $\boldsymbol{\beta}_{c}\left(t_{i j}\right)$ by $\boldsymbol{\beta}_{c}\left(t_{0}\right)$ and $\sigma_{c}^{* 2}\left(t_{i j}\right)$ by $\sigma_{c}^{* 2}\left(t_{0}\right)$ for $t_{i j}$ in the neighborhood 
of $t_{0}$. Thus, the corresponding local log-likelihood function is

$$
\sum_{i=1}^{n} \sum_{c=1}^{C} r_{i c}^{(l+1)} \sum_{j=1}^{N_{i}}\left[\log \phi\left\{y_{i j} \mid X_{i}\left(t_{i j}\right)^{T} \boldsymbol{\beta}_{c}\left(t_{0}\right), \sigma_{c}^{* 2}\left(t_{0}\right)\right\}\right] K_{h}\left(t_{i j}-t_{0}\right),
$$

where $K_{h}(t)$ is a rescaled kernel function $h^{-1} K(t / h)$ with a kernel function $K(t)$. Maximizing (5.5) with respect to $\boldsymbol{\beta}_{c}\left(t_{0}\right)$ and $\sigma_{c}^{* 2}\left(t_{0}\right)$ yields $(2.9)$ and (2.10).

\section{References}

Aneiros-Pérez, G., Cardot, H., Estévez-Pérez, G., and Vieu, P. (2004). Maximum ozone concentration forecasting by functional non-parametric approaches. Environmetrics, 15(7):675-685.

Aneiros-Pérez, G. and Vieu, P. (2006). Semi-functional partial linear regression. Statistics \& Probability Letters, 76(11):1102-1110.

Bongiorno, E. G., Salinelli, E., Goia, A., and Vieu, P. (2014). Contributions in Infinite-Dimensional Statistics and Related Topics. Societa Editrice Esculapio.

Bosq, D. (2000). Linear Processes in Function Spaces. Springer,New York.

Bosq, D. and Blanke, D. (2007). Inference and Prediction in Large Dimensions. Wiley.

Cai, Z., Fan, J., and Li, R. (2000). Efficient estimation and inferences for varyingcoefficient models. Journal of the American Statistical Association, 95(451):888902.

Chen, D., Hall, P., Müller, H.-G., et al. (2011). Single and multiple index functional regression models with nonparametric link. The Annals of Statistics, 39(3):17201747.

Chen, H., Chen, J., and Kalbfleisch, J. D. (2001). A modified likelihood ratio test for homogeneity in finite mixture models. Journal of the Royal Statistical Society: Series B, 63(1):19-29. 
DeSarbo, W. S. and Cron, W. L. (1988). A maximum likelihood methodology for clusterwise linear regression. Journal of classification, 5(2):249-282.

Ettinger, B., Guillas, S., and Lai, M.-J. (2012). Bivariate splines for ozone concentration forecasting. Environmetrics, 23(4):317-328.

Eubank, R., Huang, C., Maldonado, Y. M., Wang, N., Wang, S., and Buchanan, R. (2004). Smoothing spline estimation in varying-coefficient models. Journal of the Royal Statistical Society: Series B, 66(3):653-667.

Fan, J. and Gijbels, I. (1996). Local polynomial modelling and its applications. Chapman, Hall, London.

Fan, J., Zhang, C., and Zhang, J. (1999). Sieve likelihood ratio statistics and wilks phenomenon. Department of Statistics, UCLA.

Fan, J., Zhang, C., and Zhang, J. (2001). Generalized likelihood ratio statistics and wilks phenomenon. The Annals of Statistics, 29(1):153-193.

Fan, J. and Zhang, J.-T. (2000). Two-step estimation of functional linear models with applications to longitudinal data. Journal of the Royal Statistical Society: Series B, 62(2):303-322.

Ferraty, F., Goia, A., Salinelli, E., and Vieu, P. (2013). Functional projection pursuit regression. Test, 22(2):293-320.

Ferraty, F. and Romain, Y. (2011). The Oxford Handbook of Functional Data Analaysis. Oxford University Press.

Ferraty, F., Van Keilegom, I., and Vieu, P. (2012). Regression when both response and predictor are functions. Journal of Multivariate Analysis, 109:10-28.

Ferraty, F. and Vieu, P. (2006). Nonparametric Functional Data Analysis: Theory and Practice. Springer. 
Frühwirth-Schnatter, S. (2006). Finite Mixture and Markov Switching Models. Springer.

Garnaut, R., Howes, S., Jotzo, F., and Sheehan, P. (2008). Emissions in the platinum age: the implications of rapid development for climate-change mitigation. Oxford Review of Economic Policy, 24(2):377-401.

Hartigan, J. (1985). A failure of likelihood asymptotics for normal mixtures. In Proceedings of the Berkeley Conference in Honor of Jerzy Neyman and Jack Kiefer,eds.L.Le Cam and R.A.Olshen,Belmont,CA: Wadsworth, 2:807-810.

Heard, N., Holmes, C., and Stephens, D. (2006). A quantitative study of gene regulation involved in the immune response of anopheline mosquitoes. Journal of the American Statistical Association, 101(473):18-29.

Hennig, C. (2000). Identifiablity of models for clusterwise linear regression. Journal of Classification, 17(2):273-296.

Hoover, D. R., Rice, J. A., Wu, C. O., and Yang, L.-P. (1998). Nonparametric smoothing estimates of time-varying coefficient models with longitudinal data. Biometrika, 85(4):809-822.

Horváth, L. and Kokoszka, P. (2012). Inference for functional data with applications, volume 200. Springer Science \& Business Media.

Hoshikawa, T. (2013). Mixture regression for observational data, with application to functional regression models. http://arxiv.org/abs/1307.0170.

Huang, M., Li, R., Wang, H., and Yao, W. (2014). Estimating mixture of gaussian processes by kernel smoothing. Journal of Business $\&$ Economic Statistics, $32(2): 259-270$. 
Huang, M. and Yao, W. (2012). Mixture of regression models with varying mixing proportions: a semiparametric approach. Journal of the American Statistical Association, 107(498):711-724.

James, G. and Sugar, C. (2003). Clustering for sparsely sampled functional data. Journal of the American Statistical Association, 98(462):397-408.

Kudraszow, N. L. and Vieu, P. (2013). Uniform consistency of knn regressors for functional variables. Statistics \& Probability Letters, 83(8):1863-1870.

Leroux, B. G. (1992). Consistent estimation of a mixing distribution. The Annals of Statistics, 20(3):1350-1360.

Li, P. and Chen, J. (2010). Testing the order of a finite mixture. Journal of the American Statistical Association, 105(491).

Liang, K.-Y. and Zeger, S. L. (1986). Longitudinal data analysis using generalized linear models. Biometrika, pages 13-22.

Lin, X. and Carroll, R. (2000). Nonparametric function estimation for clustered data when the predictor is measured without/with error. Journal of the American Statistical Association, pages 520-534.

Lu, Z. and Song, X. (2012). Finite mixture varying coefficient models for analyzing longitudinal heterogenous data. Statistics in Medicine, 31(6):544-560.

Luan, Y. and Li, H. (2003). Clustering of time-course gene expression data using a mixed-effects model with b-splines. Bioinformatics, 19(4):474-482.

Ma, P. and Zhong, W. (2008). Penalized clustering of large-scale functional data with multiple covariates. Journal of the American Statistical Association, 103(482):625636. 
Marron, J. S. (1988). Automatic smoothing parameter selection: a survey. Empirical Economics, 13(3-4):187-208.

McLachlan, G. and Peel, D. (2000). Finite Mixture Models, volume 299. John Wiley \& Sons.

Ramsay, J. O. and Silverman, B. W. (2002). Applied Functional Data Analysis, volume 77. Springer, New York.

Ramsay, J. O. and Silverman, B. W. (2005). Functional Data Analysis. Springer, New York.

Rice, J. and Silverman, B. (1991). Estimating the mean and covariance structure nonparametrically when the data are curves. Journal of the Royal Statistical Society. Series B, pages 233-243.

Sapatnekar, S. S. (2011). Overcoming variations in nanometer-scale technologies. Emerging and Selected Topics in Circuits and Systems, IEEE Journal on, 1(1):518.

Titterington, D., Smith, A., Makov, U., et al. (1985). Statistical Analysis of Finite Mixture Distributions. Wiley, New York.

Verbeke, G. and Lesaffre, E. (1996). A linear mixed-effects model with heterogeneity in the random-effects population. Journal of the American Statistical Association, 91(433):217-221.

Xu, W. and Hedeker, D. (2001). A random-effects mixture model for classifying treatment response in longitudinal clinical trials. Journal of Biopharmaceutical Statistics, 11(4):253-273.

Yakowitz, S. and Spragins, J. (1968). On the identifiability of finite mixtures. The Annals of Mathematical Statistics, 39(1):209-214. 
Yao, F., Fu, Y., and Lee, T. C. (2011). Functional mixture regression. Biostatistics, $12(2): 341-353$.

Yao, F., Müller, H., and Wang, J. (2005). Functional data analysis for sparse longitudinal data. Journal of the American Statistical Association, 100(470):577-590.

Young, D., Hunter, D., Elmore, R., Xuan, F., Hettmansperger, T., and Thomas, H. (2007). The mixtools package: tools for mixture models. R Package Version 0.2.0. 